\title{
Editorial: Scientific advances in product experience engineering
}

\author{
Cheng-Hung Lo ${ }^{1}$. Chih-Hsing $\mathrm{Chu}^{2}$ - Hideyoshi Yanagisawa ${ }^{3}$. \\ Jianxin (Roger) $\mathrm{Jiao}^{4}$
}

Received: 15 August 2016/ Accepted: 14 September 2016 / Published online: 23 September 2016

(C) Springer Science+Business Media New York 2016

\section{Need-driven experience study}

Abraham Maslow, a humanistic psychologist, studied human needs and developed one of the most articulated and accepted theories about them-Maslow's hierarchy of needs (Fig. 1). In his theory, human needs are divided into five layers (Maslow 1970).

- Physiological: Very basic survival needs for humans' physiological functions (e.g., food, water, air, etcetera).

- Safety: The need to stay in a safe environment and interact safely with the elements in that environment

- Love/Belonging: The need to find a life partner as well as to belong to a group or society (e.g., family and other agencies of social support)

- Esteem: The need to be respected as an individual

- Self-actualization: The need to become a complete self

$\triangle$ Chih-Hsing Chu

chchu@ie.nthu.edu.tw

Cheng-Hung Lo

Ch.Lo@xjtlu.edu.cn

Hideyoshi Yanagisawa

hide@mail.design.t.u-tokyo.ac.jp

Jianxin (Roger) Jiao

rjiao@gatech.edu

1 Department of Industrial Design, Xi' an Jiaotong-Liverpool University, Suzhou, China

2 Department of Industrial Engineering and Engineering Management, National Tsing Hua University, Hsinchu, Taiwan

3 Department of Mechanical Engineering, The University of Tokyo, Tokyo, Japan

4 Department of Mechanical Engineering, Georgia Institute of Technology, Atlanta, GA, USA
Maslow further elaborated about how people pursue the layer of needs progressively. One will, first, fulfil physiological needs in order to survive. Then, he or she will demand the assurance of personal safety. Then comes the need to feel love or a sense of belonging to a social group. What follows is the need to learn about oneself, appreciate the uniqueness, and be respectful toward others and their individuality. The ultimate need is to grow and reach one's full potential.

If we recall the focuses of ergonomics and recent studies about user experience (UX) against this hierarchy of needs, it is interesting to see how they correspond to the different levels of needs. One could say that ergonomics focuses on the first two layers of needs in Maslow's hierarchy, as the discipline highlights human physiological responses and constraints so that a machine, product, or system could be built toward fulfilling these functional needs. Then, human operator can work with the products in a safe, error-proof, learnable, and efficient way. On the other hand, UX studies seem to correspond to satisfying needs in the upper layers. As manufacturing sites continue to populate globally in the process of industrialization, rarely does a company release a consumer product that no other competitors can produce. The manufacturing technique is no longer the dominant factor for deciding whether a product is successful. More and more product users seek design features or attributes that respond to their intangible, so-called emotional needs. It is not difficult to understand why modern UX studies focus on aspects like pleasurability, aesthetics, affectivity, personalization, and social recognition, among others.

\section{Product experience engineering}

From scientific and engineering perspectives, ergonomics measures and analyzes human factors and develops design 


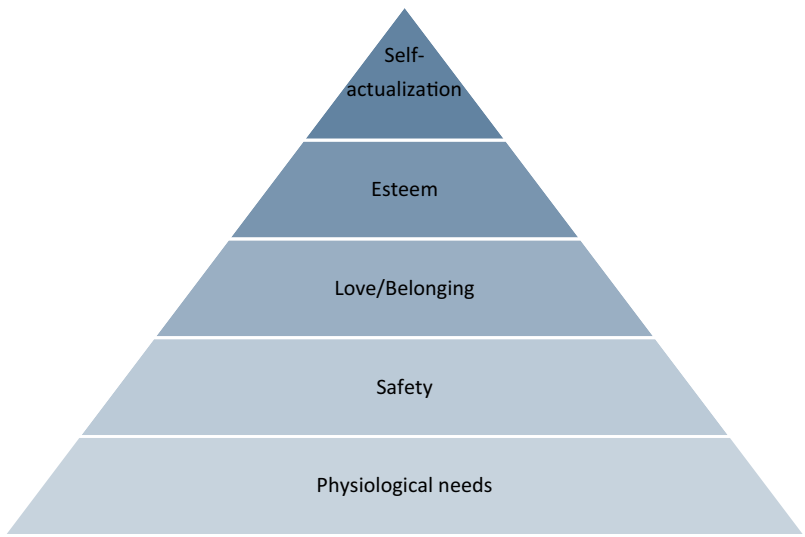

Fig. 1 Five layers of human needs

requirements (McCauley-Bush 2011). However, most UX studies, probably because of the many intangible aspects involved, often generate qualitative design insights or guidelines. A challenge still remains in efficiently translating the findings to product design specifications. Product designers or engineers often rely on their own interpretations of UX-oriented insights and guidelines, which may lead to caseby-case, non-replicable design results. This is not to say that every aspect of UX can be measured and converted into quantitative specifications. However, more scientific methods for studying human behaviors, such as those developed in psychology or cognitive science, can be incorporated to measure UX. Indeed, related fields exist, including cognitive ergonomics (McCauley-Bush 2011), emotional/affective design (Lo and Chu 2009; Lo et al. 2015), and user modeling (Kobsa 2001), to name but a few that already contribute to the scientific understanding of UX issues.

Addressing UX according to a holistic approach drives many recent innovations in the design industries (Brown 2009). Taking the far end of the UX spectrum to fully satisfy user needs may not be feasible under many circumstances; the cost to rebuild everything from scratch is just one consideration. It is also important to reflect on what a company or manufacturer has already established. The solid foundation of a company responsible for its unique value proposition, along with the component base and production pipelines, are valuable resources to support innovation. Indeed, innovation is more about efficient transition and integration of technologies to accommodate new market or user demands, building on top of what the company or manufacturer already has at hand.

Taking the above factors into account, a conceptual framework, product experience engineering (PEE), emerged with our call for contributions for this special issue. It is essentially a process of transforming UX to design and manufacturing decisions. The conceptual framework consists of four facets: Measurement and Analytics, Cognitive Mapping, Design

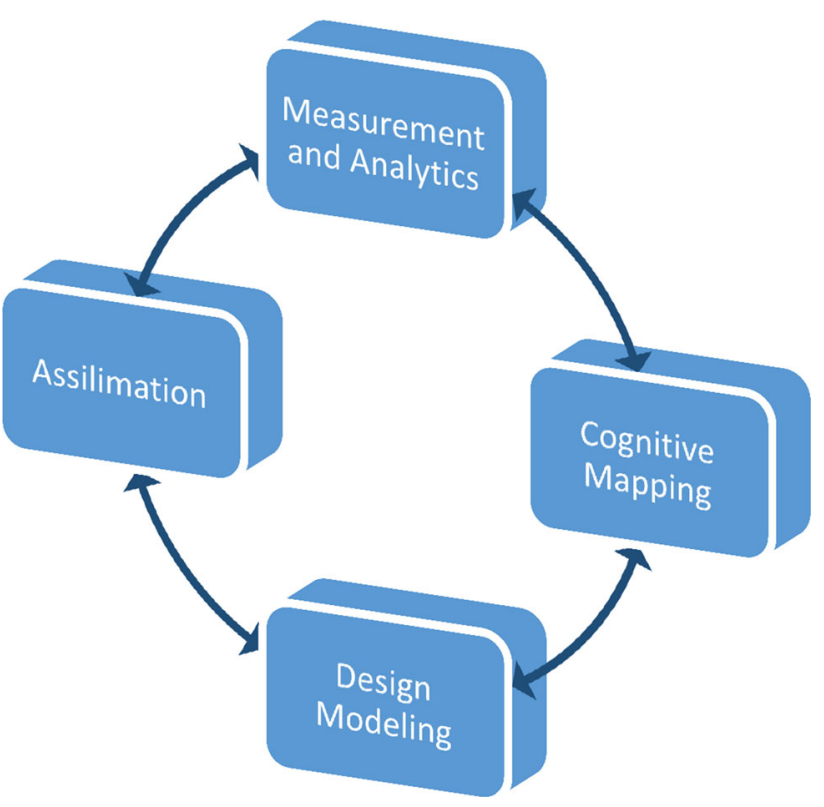

Fig. 2 A proposed framework for Product Experience Engineering (PEE)

Modeling, and Assimilation (see Fig. 2). These four facets will be discussed further, together with the collected works in the following section.

On one hand, PEE distinguishes itself from conventional ergonomics practices by including emotional/affective factors that constitute an important part of UX. On the other hand, it provides design and engineering solutions that support the experiential inputs. We hope the collected works can contribute to building a scientific foundation, which will provide a more systematic connection between $\mathrm{UX}$ and product design methods.

\section{PEE facets and the collected works}

\section{Measurement and analytics}

One of the user factors emphasized in recent UX studies is the emotional responses of users toward a design. The observable changes in human behaviors resulted from changes in emotional states. Of course, the physiological indices (e.g., heart rate, pupil size, and brain wave) were also correlated. Emotional changes were manifested through these physiological means. Thanks to the development of sensor technology, these emotionally driven physiological signals can be monitored and captured more conveniently. They provide more scientific and precise measures of a user's experience when interacting with a product. In the first paper, Balter and Steinez provide a systemic view on how to utilize these sensor technologies for investigating UX and how the captured results may be used for engi- 
neering design and relevant applications. In Nguyen and Zeng's experimental study, although targeted on design activities, can be applied to evaluate purposeful experiences, such as completing a functional task with a product.

The experimental approach focuses on individual experiences. In the near future, UX studied on a mass scale will become increasingly more important in guiding the design of products. Customers' feedback, users' issues with products, and maintenance requests are collected online nowadays. These various data can be valuable resources to understand how a product performs in the market; data also provide information about a product's UX. One of the challenges is how to represent this massive collection of user data for human comprehension. In the third paper, Adagha and Levy systematically reviewed the recent development of visual analytics, a discipline that has grown rapidly with the recent trend of dealing with big data. Researchers in this field seek ways to transform data into visual representation. Representations elaborate on the results of data analysis as well as on intermediate outcomes that facilitate further exploration and understanding of user data.

\section{Cognitive mapping}

Models that map empirical findings to behavioral or design patterns (e.g., decision making process or cognitive modeling)

One of the key challenges to studying UX is finding appropriate quantitative measures. Thanks to the recent study regarding the use of mathematical models to represent human behaviors, we are allowed to provide a mathematical description of repeated, patterned human behaviors. In the fourth paper, Yanagisawa and Takatsuji model the expectation effects of customers using information theory. This approach may lead to more effective design patterns.

Without a doubt, many aspects of UX would be better understood with a qualitative approach. Indeed, we have started to see some recent works in engineering that integrate qualitative measures (Segel and Heer 2010) or inquiry methods (Dekker et al. 2003) to guide the design of physical or digital artefacts. Analepsis, or flashbacks, which originated from theatrical practice, is a technique to refresh the audience's memory to facilitate storytelling in plays or films. In the fifth paper by Tsao and Chen, the authors argued that the same technique may be used to construct a mental image of the experience after using a product. Moreover, that mental image may represent the overall impression of that experience. The mental image can then be used to inform designers and even trigger development of design guidelines.

\section{Design modeling}

Design features or components are integrated so that they can be parameterized by user responses or preferences.

In the sixth paper by Wu and Akgunduz, although the target audience is physically-disabled users, this work presents the possibility of using bio-signals for modeling geometries. It demonstrates the potential to exhibit user preferences through observable changes in a product's form or shape. From engineering design and manufacturing perspectives, accommodating user preferences is like customizing products on a massive scale. To facilitate mass customization, novel CAD techniques are needed to deal with component assembly to generate products constructed with customized features. In particular, intelligent ways to query and retrieve from a product component database are important. Nguyen and Zeng investigated the relationships between subjective rating measure and physiological measure of mental stress and mental effort during design activities. A strong association between self-rated effort and EEG signals was found in several design tasks. They suggested that studies based on subjective rating should take into account the stress and effort of respondents during the rating activities to ensure the validity of the self-report. In the seventh paper, Chu et al. utilized human judgments regarding shape similarity and proposed a 3D geometrical approach to search the needed components. One of the often practiced approaches for understanding product experience is generating product prototypes for users. User feedback can then be used as guiding knowledge to refine product design. However, producing physical prototypes results in extra cost of development, which may not be feasible for companies on a smaller scale. To study $\mathrm{UX}$, the cost to produce a working prototype could be a huge burden to a company. However, the eighth paper of Ferrise et al. introduced virtual reality (VR) technology, which facilitates the evaluation of prototypes by presenting alternatives through multisensory content for a more holistic evaluation.

\section{Assimilation}

This facet refers to the phase when PEE is put into real-world practice. We call it assimilation rather than implementation because we hope PEE can be adaptable and developed continuously through industrial and business practices. The obstacles and challenges during implementation and their practical solutions should be deemed as valuable information for augmenting PEE. It is our intention to determine whether the PEE framework can be applied in a more holistic scenario. In this issue, we have included three practical or case-oriented studies. In the ninth paper, Yadav at al. proposed an integrative design approach combining the Kano model, Taguchi method, and Grey Relation Analysis (GRA) to obtain the optimal combination of shape parameters and 
aesthetic aspects for a car profile. In the tenth paper, Hyun et al. identified correlations of design intent and user response to stylistic recognition of 23 car brands, with an emphasis on visual aesthetics. The distributions of the typical design elements and novel design elements were found by evaluating car exterior designs based on shape similarities. The result was compared with looking probabilities on design elements observed from eye tracking experiments. The experimental findings indicate that creating unique designs may not always be good strategies for improving design qualities, brand recognition or even purchase intent. In the next paper, Lin and Cheng proposed a conceptual UX interaction model that incorporates the characteristics of usability and user interaction level based on quality of experience. A quantitative survey for mobile phone users was developed to investigate differences among types of user experience and product characteristics.

\section{Epilogue}

It is imperative to develop mathematical and computational models for eliciting, quantifying, evaluating, and reasoning affective-cognitive needs inherent in the fulfillment of user experience. The last study by Jiao et al. discusses the key research issues for understanding how human users' subjective experience and affective prediction impact their choice behavior under uncertainty. To echo the framework shown in Fig. 2, the authors proposed a conceptual framework by extending prospect theory in the field of behavioral economics to the modeling of choice behavior in user experience. The paper also highlights the promise of setting up user experiments to predict user affects through information extraction of augmented user experience via multimodal physiological signals and motion tracking and sensing.

We would like to express our sincere gratitude to the editing and publishing team of the Journal of Intelligent Manufacturing. The journal is a well-established publishing outlet for engineering researchers. We hope this special issue will extend and strengthen the journal's scope on UX studies. We also appreciate the tremendous help provided by the following reviewers during the review process: Jun-Ming Lu, Cheng-Hung Lo, Marianella Chamorro-Koc, Peng-Cheng Sung, Jan Bernhard deMeer, Hideyoshi Yanagisawa, Ji-Hyun Lee, Martin Steinert, Chao-Yang Yang, Kazutaka Ueda, Katja Holtta-Otto, Cheng-Jhe Lin, TLim, Shana Smith, Hsin-Chieh Wu, Caterina Rizzi, Chaiwoo Lee, Rajeev Jain, Sangwoo Bahn, S.C. Johnson Lim, Yong Zeng, Yuan-Chi Tseng, MingChuan Chiu, Serena Graziosi, Chiuhsiang Joe Lin, Chih H. Wang, Tsai Chi Kuo, Hwann-Tzong Chen, Szu-Hao Huang, Feng Zhou, Pisut Koomsap, Jari Varsaluoma, Chih-Hsing Chu, Qianli Xu, Hiroshi Hashimoto, Konstantinos Trochidis, Tamotsu Murakami.

\section{References}

Brown, T. (2009). Change by design: How design thinking transforms organizations and inspires innovation. New York: Harper Business.

Dekker, S. W. A., Nyce, J. M., \& Hoffman, R. R. (2003). From contextual inquiry to designable futures: What do we need to get there? IEEE Intelligent Systems, 18, 74-77.

Kobsa, A. (2001). Generic user modeling systems. User Modeling and User-Adapted Interaction, 11, 49-63.

Lo, C. H., \& Chu, C. H. (2009). Affective modelling: Profiling geometrical models with human emotional responses. Computer Graphics Forum, 28, 1811-1820.

Lo, C. H., Chu, C. H., \& Huang, S. H. (2015). Evaluating the effect of interactions between appearance-related product designs and facial characteristics on social affectivity. International Journal of Industrial Ergonomics, 45, 35-47.

Maslow, A. H. (1970). Motivation and Personality (2nd ed.). New York: Harper \& Row.

McCauley-Bush, P. (2011). Ergonomics: Foundational principles, applications, and technologies. Boca Raton: CRC Press.

Segel, E., \& Heer, J. (2010). Narrative visualization: Telling stories with data. IEEE Transactions on Visualization and Computer Graphics, 16, 1139-1148. 